\title{
Design of a Compact Gaussian Profiled Corrugated Horn Antenna for Low Sidelobe-Level Applications
}

\author{
Tohid Salimi, Amir Maghoul, and Ali A. Abbasid
}

\begin{abstract}
In this paper, the design of a circular corrugated horn antenna is presented for low sidelobe-level. The basic requirement of this design is compactness. Therefore, we use corrugated Gaussian profiled horn antennas to improve the size and sidelobe level simultaneously. Because of compact design of this horn, it is not expected to have a good symmetry in $\mathbf{E}$ and $\mathrm{H}$ planes. The obtained radiation patterns are sufficient for desired applications. The proposed corrugated antenna has a longitudinal section composed of a normal corrugated horn, some straight corrugations, and a Gaussian profile to complete transition between waveguide and the free space.
\end{abstract}

Index Terms - Corrugated horn, compactness, gaussian profiled, sidelobe-level.

\section{INTRODUCTION}

High-performance microwave satellite communications, radar and remote sensing systems often use a reflector antenna as end component of the transmitter and receiver front ends. A reflector antenna consists of two parts: the first one is the reflector itself and the second one is the horn at the focus. The horn antenna is a crucial component because it must match the microwave signal from the source to the reflector antenna with the minimum losses and the maximum efficiency. There are a variety of possible designs for this horn, but the preferred choice at the present for high-performance systems is the corrugated horn [1].

Directivity, gain, sidelobe and cross-polarization levels are important design parameters for many applications involving horn antennas. Additional design parameters, relevant to satellite applications are length and weight, which need to be minimized [2], [3].

The main goal of horn antenna in the last years was to excite a well-known $\mathrm{HE}_{11}$ mode from proper mixture of $85 \%$ of $\mathrm{TE}_{11}$ mode, and $15 \%$ of $\mathrm{TM}_{11}$ mode, with correct phase to produce a Gaussian like radiation pattern. This mode corresponds to the fundamental mode of a circular corrugated waveguide [4], [5]. This technique reported in [6]-[9], involves a gradual matching of the smooth monomode circular waveguide to another corrugated one wherein the corrugation depth is smoothly tapered from $1 / 2$ to $1 / 4$ of a wavelength, that is shown in Fig. 1.

The Gaussian (also called hyperbolic) profile is very

Manuscript received September 12, 2012; revised November 22, 2012.

T. Salimi is with the Communication Engineering Department, Space Research center of Iran, Tehran, Iran (e-mail: salimitohid@gmail.com)

A. Maghoul is with the EMC department in space research center of Iran, Tehran, Iran (e-mail: amir.maghoul@yahoo.com).

A. A. Abbasi is with the Antenna Department, Space Research center of Iran, Tehran, Iran (e-mail: aaaara@yahoo.com) useful for completing the transition of the horn flare from the mid-range matching section to the aperture. Also it results in a compact horns compared with a linear taper [10].

There are three main reasons for the existence of corrugated horn antennas. Firstly, they exhibit radiation pattern symmetry, which offers the potential for producing reflector antennas with high gain and low spillover; secondly, they radiate with very low cross-polarization, which is essential in dual polarization systems and finally, they offer a wide bandwidth responses.

Corrugated GPHA's are better than the rest of corrugated horn antennas in two particular aspects:

1) They present much lower sidelobes than any other corrugated profile.

2) They are usually shorter than the rest of corrugated profiles because their performance qualities are superior [11].

\section{Design PRocedure}

\section{A. $T E_{11}$ to $H E_{11}$ Mode Converters}

Gaussian profiled horn antenna (GPHA) needs to be fed by a quite pure $\mathrm{HE}_{11}$ mode. We should select which type of $\mathrm{TE}_{11}$ to $\mathrm{HE}_{11}$ converter is suitable for feeding a Gaussian Profiled antenna.

This mode converter usually starts from a smooth circular monomode waveguide propagating the $\mathrm{TE}_{11}$ mode and ends at the required aperture diameter to feed the corrugated GPHA model. One could find several types of $\mathrm{TE}_{11}$ to $\mathrm{HE}_{11}$ mode converters. One of them is the horn type proposed by Potter; its disadvantage is a poor bandwidth. Another type of $\mathrm{TE}_{11}$ to $\mathrm{HE}_{11}$ mode converter could be just a conical corrugated horn antenna, its disadvantage is its size. Another corrugated GPHA can be another possibility of $\mathrm{TE}_{11}$ to $\mathrm{HE}_{11}$ converter with the same disadvantage of a conical corrugated one but with slightly better bandwidth characteristics [4]. It is well known that a choked antenna offers one of the shortest antenna profiles with rather good radiation features [12]. This antenna exhibits two principal disadvantages: a narrow bandwidth and it is very sensitive to manufacturing tolerances in the throat region (mode generator) [13]. We choose the normal corrugated horn antenna as a $\mathrm{TE}_{11}$ to $\mathrm{HE}_{11}$ converter.

Design frequency band is from $10.5 \mathrm{GHz}$ to $14.5 \mathrm{GHz}$. The proposed conical corrugated horn design parameters as shown in Fig. 1 , are $\theta_{f}=23.3^{\circ}, w=3.7 \mathrm{~mm}, t=1 \mathrm{~mm}, p=4.7$, and $2 a=24 \mathrm{~mm}$, with depths changing from $\lambda / 2$ to $\lambda / 4$ smoothly. The $\lambda$ is the wavelength at center frequency. 


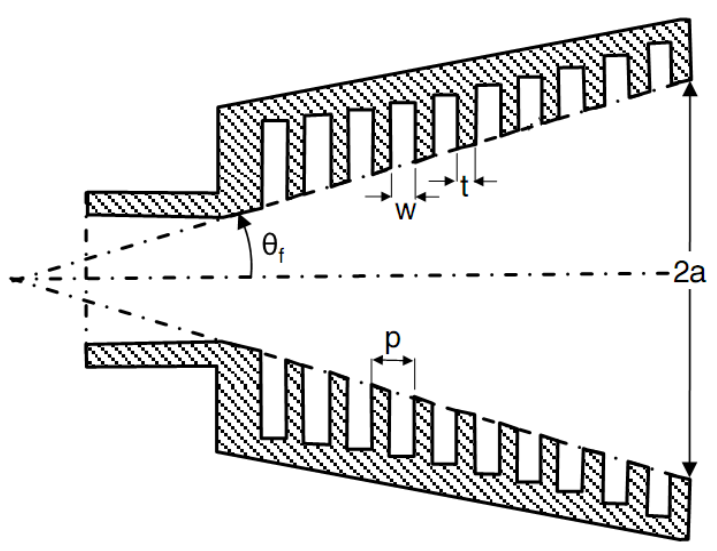

Fig. 1. Normal corrugated horn with design parameters has showed [8].

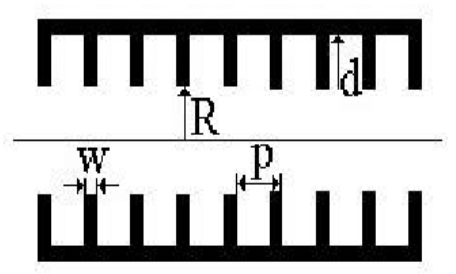

Fig. 2. Phasing section with corrugations

\section{B. Phasing Section}

Because of compactness of corrugated horn antenna in first section, directivity is low and phase centers on $\mathrm{E}$ and $\mathrm{H}$ planes are not coincident. So we use a phasing section to place phase centers together in the desired frequency band. Also it improves the mixture of $\mathrm{TE}_{11}$ and $\mathrm{TM}_{11}$ modes. Actually when this section is attached to the normal corrugated horn, they result in a potter like horn with new characteristics in bandwidth and radiation pattern (We named it potter corrugated horn). The phasing section is shown in Fig. 2 and design parameters is $w=3.7 \mathrm{~mm}, p=4.7 \mathrm{~mm}$, and $d=7.3 \mathrm{~mm}$.

\section{Gaussian Profiled Horn Antenna (GPHA)}

It is well known that one of the best ways to define a free space radiation from an antenna is by means of the paraxial free space modes, the Gaussian modes, which are a solution of the paraxial free space equation. A complete theory about properties of Gaussian modes is not presented here. The main mode for our corrugated horn radiation purposes is the fundamental Gaussian mode. This mode has null cross-polarization and null sidelobes. Its electric field propagation formula can be expressed as follows:

$$
E(r, \varphi, z)=\frac{w_{0}}{w(z)} \cdot \exp ^{\frac{-r^{2}}{w^{2}(z)}} \cdot \exp ^{-j \frac{k r^{2}}{2 \cdot R(z)}} \cdot \exp ^{-j(k z-\xi(z))}
$$

where $r^{2}=x^{2}+y^{2}$ and $w(z)$ is the beamwidth where there is a field decay of $1 / e$ respect to the maximum, (see Fig. 3). In $z=$ 0 , the function $w(z)$, (see (2)), has a minimum known as beamwaist and called $w_{0}$.

$$
w(z)=w_{0} \cdot \sqrt{1+\left(\frac{2 . z}{k w_{0}^{2}}\right)^{2}}
$$

where $w_{0}=\alpha . r_{0}=\alpha . D_{0} / 2$ is the beamwaist at $z=0$ related with the $\mathrm{D}_{0}$ through the parameter $\alpha$. The $\alpha$ parameter controls the aperture angle of the horn for a given frequency and waveguide radius, and $k=2 \pi / \lambda$ is the wavenumber in free space. The corresponding waveguide profile which follows the curve for Gaussian equi-amplitude relative surface is given by:

$$
R(z)=r_{0} \cdot \sqrt{1+\left(\frac{2 \cdot z}{k \cdot \alpha^{2} \cdot r_{0}^{2}}\right)^{2}}
$$

Fig. 4 shows the relationship between the horn profile (3) and the beamwaist propagation imposed by (2). The $\alpha$ parameter can vary between 0.5 and 0.8 but usually the optimum value is around 0.65 . The last parameter we must define to completely design the corrugated GPHA is the profile length. If the antenna is very long we can say that we are over-guiding the Gaussian beam and the addition of more antenna length is not really improving the beam, so the efficiency can be the same than with a shorter antenna [11]. The best value for very nice Gaussian beam efficiency and not excessive length (which means low sidelobes) is obtained to be $33 \mathrm{~mm}$.

The design parameter of this section is $r_{0}=12 \mathrm{~mm}$, and $\alpha=0.65$. Reason for choosing 0.65 is the proper mode mixture of $\mathrm{HE}_{11}$. Designed horn is completely shown in Fig. 5 with the total length of $3.73 \lambda$ and output aperture of $3.1 \lambda$.

\section{Simulation RESUlts}

Fig. 6 shows the simulated radiation patterns of the designed corrugated horn antenna and Fig. 7 shows the simulated return loss. Fig. 6(a)-(g) shows the radiation patterns of the horn in desired and out of design frequency band. In the desired frequency band, the sidelobe level is below $-38 \mathrm{~dB}$ that is a good result. High frequency is the limitation in the sidelobe level bandwidth. As shown in Fig. 6(f), (g), the sidelobes rise at higher frequency and limit the sidelobe bandwidth to about $6 \mathrm{GHz}$. Also maximum cross-polarization level is $-28 \mathrm{~dB}$ in the frequency band of $10 \mathrm{GHz}$ to $17 \mathrm{GHz}$. Pattern symmetry is very good and $-10 \mathrm{~dB}$ beamwidth variations are about 6 degrees.

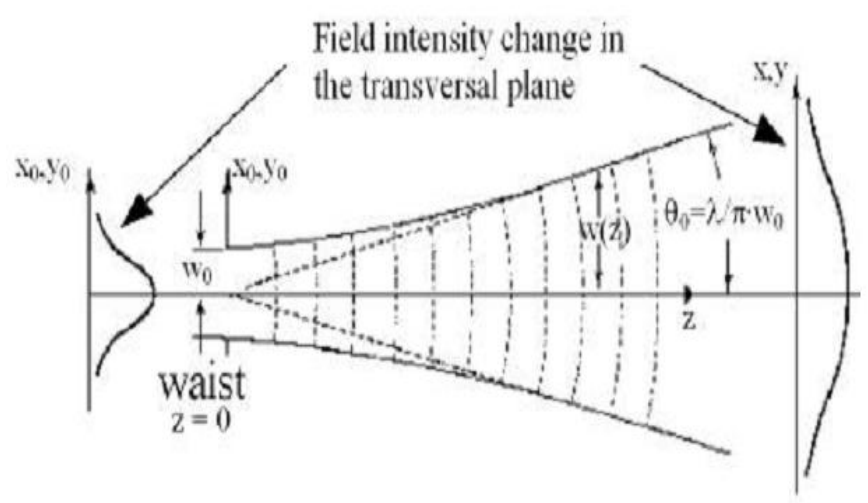

Fig. 3. Propagation of a fundamental Gaussian beam mode [13]. 


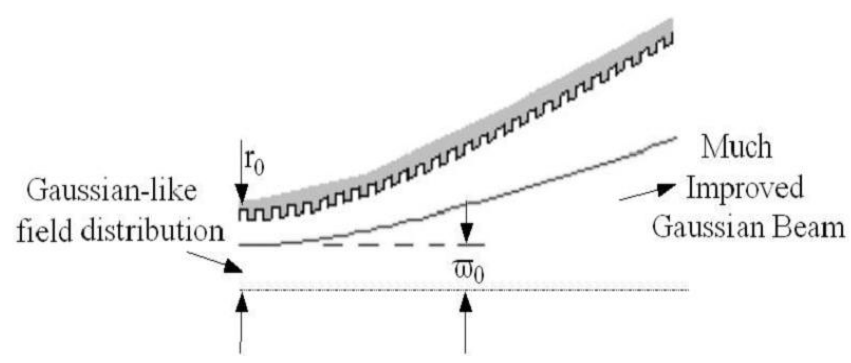

Fig. 4. Corrugated gaussian profiled horn antenna, (corrugated GPHA)[13].

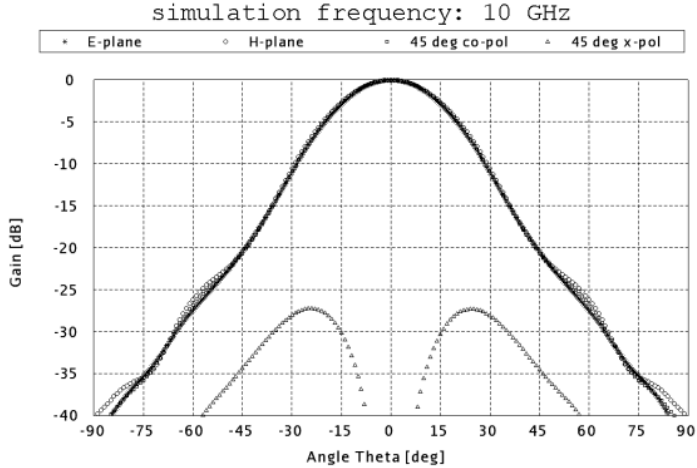

(a)

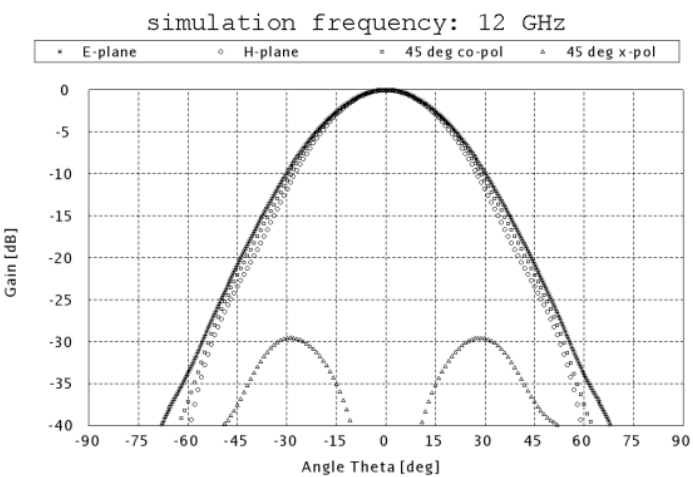

(c)

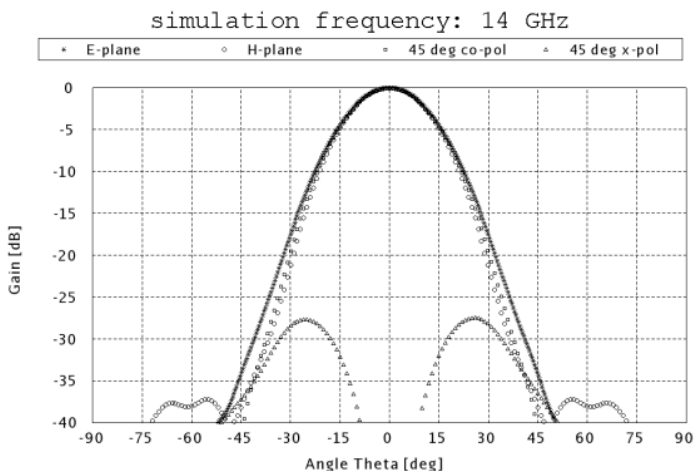

(e)

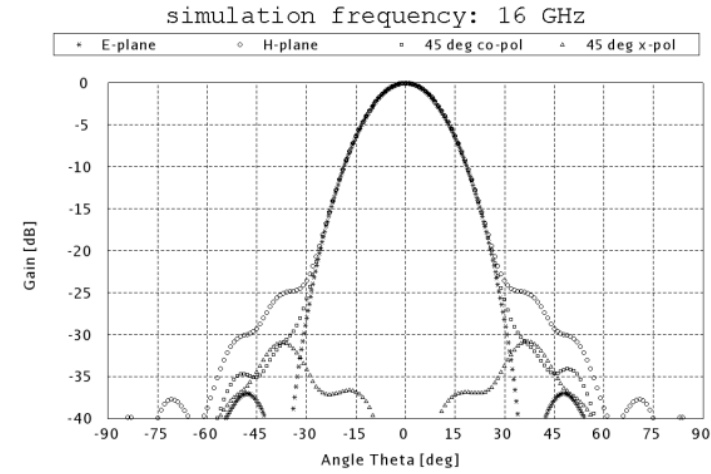

(g)

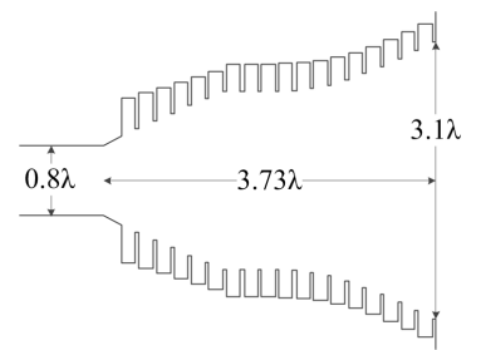

Fig. 5. proposed corrugated horn + GPHA.

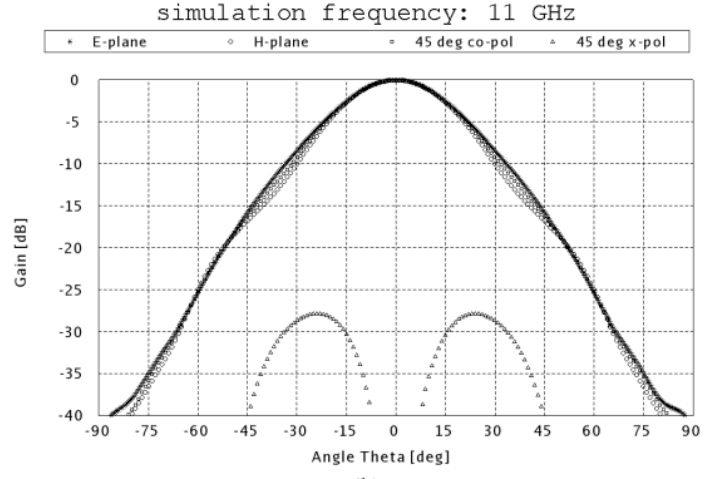

(b)

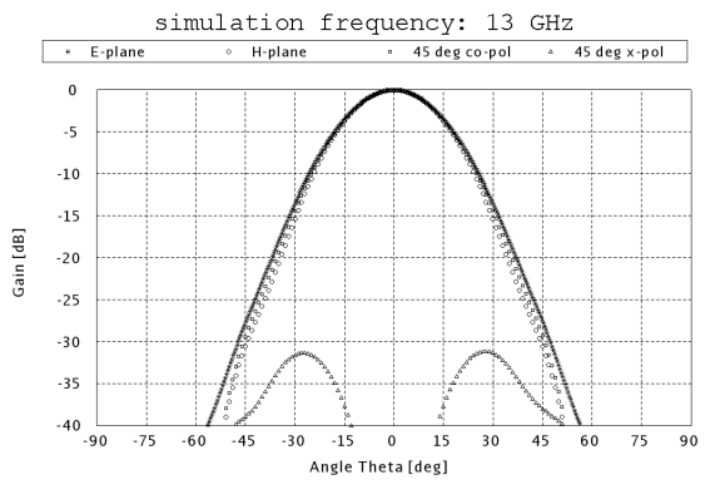

(d)

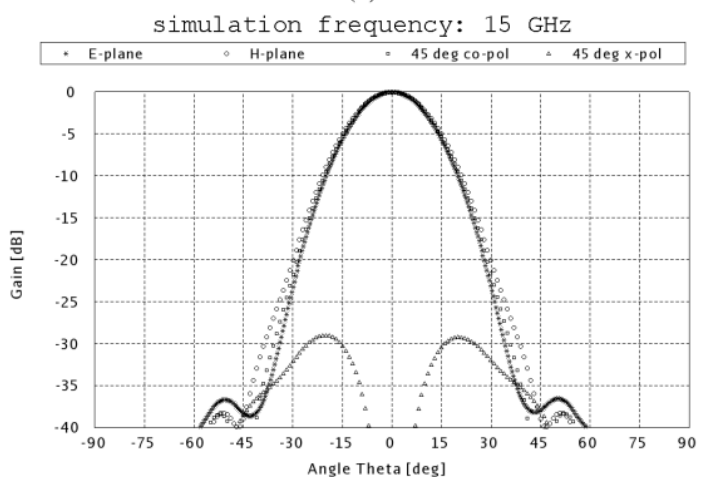

(f)

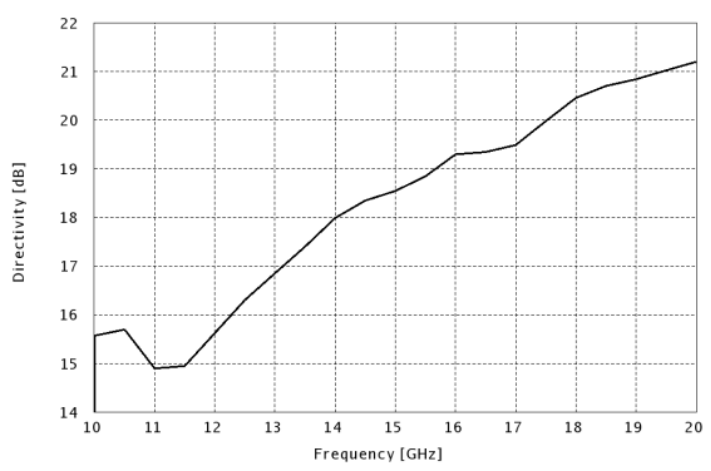

(h)

Fig. 6. Radiation characteristics of corrugated horn + GPHA. (a)-(g) gain, and (h) directivity. 


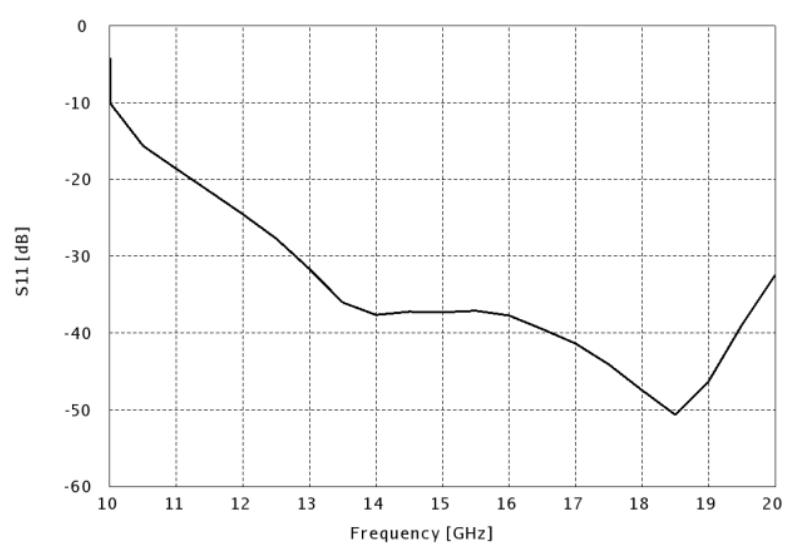

Fig. 7. Simulated return loss of corrugated horn + GPHA.

\section{CONCLUSION}

In this paper the design of a compact corrugated horn antenna for low sidelobe level application using Gaussian profiled horn antennas is presented. Advantages of GPHAs versus other corrugated horns are mentioned. the proposed antenna uses the normal corrugated horn antenna as a mode conversion from $\mathrm{TE}_{11}$ to $\mathrm{HE}_{11}$. Also it's possible to do this conversion with another GPHA to reduce the size slightly more. Also by increasing the length of the GPHA with proper feeding it by a high purity of $\mathrm{HE}_{11}$ mode, the cross-polarization level can be decreased to $-50 \mathrm{~dB}$ for the satellite applications. In these applications, there is a trade-off between size and cross-polarization level.

\section{REFERENCES}

[1] A. D. Olver, P. J. B. Clarricoats, A. A. Kishk, and L. Shafai, "Microwave horns and feeds," Chap. 8 and 9, IEEE Electromagnetics Waves Series vol. 39, 1994.

[2] G. L. James, "Design of wide-band compact corrugated horns," IEEE Transactions on Antennas and Propagation, vol. AP-32, pp. 1134-1138, 1984.

[3] C. Granet, T. S. Bird, and G. L. James, "Compact low-sidelobe corrugated horn for global earth coverage," in Proceedings of the IEEE Antennas and Propagation International Symposium and URSI Radio Science Meeting, Orlando, Florida, 11-16 July 1999, pp. 712-715.

[4] J. T. Vallinas, R. G. García, and C. D. R. Bocio, "Modern corrugated horn antenna design for extremely low sidelobe level," Electric and Electronic Engineering Department. Public University of Navarra.

[5] C. D. Río, R. Gonzalo, and M. Sorolla, "High purity guassian beam excitation by optimal horn antenna," E.T.S.I.I y Telecommunication. Public University of Navarra.
[6] P. J. B. Clarricoats and A. D. Olver, "Corrugated horns for microwave antennas," IEEE Electromagnetics Waves Series, Chap. 5, 6 and 7, Peter Peregrinus, vol. 18, 1984

[7] A. D. Olver, P. J. B. Clarricoats, A. A. Kishk, and L. Shafai, "Microwave horns and feeds," IEEE Electromagnetic Waves Series, The Institution of Electrical Engineers, vol. 39, 1994.

[8] G. L. James, "Analysis and design of TE11 to HE11 corrugated cylindrical waveguide mode converters," IEEE Transactions on Microwave Theory and Techniques, vol. MTT-29, pp. 1059-1066, 1981.

[9] B. Maca, Thomas, G. L. James, and K. J. Greene, "Design of high-performance wideband corrugated horns for Cassegrain antennas," IEEE Transactions on Antennas and Propagation, vol. AP-34, pp. 750-757, 1986.

[10] J. L. Volakis, Antenna Engineering Handbook, 4th ed. ISBN 0-07-147574-5.

[11] J. T. Vallinas, "Modern corrugated horn antennas," Telecommunication. Public University of Navarra, July 2003.

[12] A. W. Rudge, K. Milne, A. D. Olver, and P. Knight, "The handbook of antenna design," in Inst. Elect. Eng. Electromagnetic Waves Series. London, U.K.: Inst. Elect. Eng., vol. 15-16, 1982.

[13] T. S. Bird, C. Granet, and G. L. James, "Lightweight compact multi-mode corrugated horn with low sidelobes for global-earth coverage," in Proc. Antennas and Propagtion'00 Conf., Davos, Switzerland, Apr. 2000.

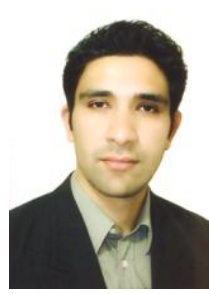

Tohid Salimi was born in Tehran, Iran, in 1985. He received his B.S. degree from the Imam Hosein University of Iran, in 2008. He is currently with Communication Engineering Department, Space Research Center of Iran, Tehran, Iran. His research has focused on Satellite Antennas, Shaped beam antennas, Earth Station Antenna Technology, Tracking Feeds, wideband Hybrids, Microwave Devices, Array antennas and Computer Networks.

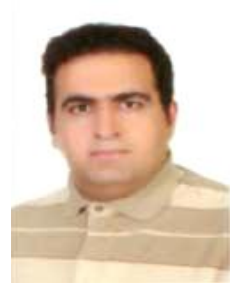

Amir Maghoul was born in Amlash, Guilan, Iran, in 1983. He received the B.Sc. degree in electrical engineering, Guilan, Iran, in 2005, the M.Sc degree in communication field and wave engineering from the University of Tabriz, Tabriz, Iran, in 2008. His research interests include Electromagnetic Compatibility (EMC), radar systems design, and antenna design. Mr. Maghoul is currently a researcher with EMC department in space research center of Iran.

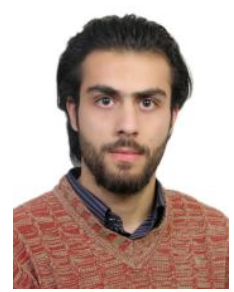

Ali Ahmad Abbasi was born in Tehran, Iran, in 1985 He received his B.S. degree from the Imam Hosein University of Iran, in 2008. He is currently with Antenna Department, Space Research Center of Iran, Tehran, Iran. His research has focused on reflector antenna design and feeding, Microstrip Antennas, Array Antenna, and Microwave Devices. 